\title{
Acute Whole-Body Vibration Exercise Promotes Favorable Handgrip Neuromuscular Modifications in Rheumatoid Arthritis: A Cross-Over Randomized Clinical
}

\author{
Ana Carolina Coelho-Oliveira $\mathbb{D}^{1,2,3,4}$ Ana Cristina Rodrigues Lacerda $\mathbb{D},,^{1,2,3}$ \\ Ana Lúcia Cristino de Souza $\left(1,{ }^{1}\right.$ Luciana Martins de Mello Santos $\left(1,{ }^{1,2,3}\right.$ \\ Sueli Ferreira da Fonseca $\mathbb{D}^{1,3}$ Jousielle Márcia dos Santos $\mathbb{D},{ }^{1,3}$ \\ Vanessa Gonçalves César Ribeiro $\mathbb{D}^{1},{ }^{1,3}$ Hércules Ribeiro Leite ${ }^{(D)},{ }^{1,2,3}$ \\ Pedro Henrique Scheidt Figueiredo $\mathbb{D}^{1,2,3}$ José Sebastião Cunha Fernandes $\left(\mathbb{D},{ }^{5}\right.$ \\ Fábio Martins $\mathbb{D},{ }^{1,2,3}$ Renato Guilherme Trede Filho $\mathbb{D}^{1,2,3}$ Mario Bernardo-Filho ${ }^{1 D},{ }^{4}$ \\ Danúbia da Cunha de Sá-Caputo $\mathbb{D}^{4},{ }^{4}$ Alessandro Sartorio $\mathbb{D D}^{6}{ }^{6}$ Darryl Cochrane $\mathbb{D}^{7}{ }^{7}$ \\ Vanessa Pereira Lima $\mathbb{D}^{1,2,3}$ Henrique Silveira Costa ${ }^{1}{ }^{1,2,3}$ \\ Vanessa Amaral Mendonça $\mathbb{D}^{1,2,3}$ and Redha Taiar $\mathbb{D}^{8}$ \\ ${ }^{1}$ Centro Integrado de Pós-Graduação e Pesquisa em Saúde (CIPq-Saúde), Universidade Federal dos Vales do Jequitinhonha e \\ Mucuri (UFVJM), Diamantina, Minas Gerais, Brazil \\ ${ }^{2}$ Programa de Pós-Graduação em Reabilitação e Desempenho Funcional (PPGReab), Universidade Federal dos Vales do \\ Jequitinhonha e Mucuri (UFVJM), Diamantina, Minas Gerais, Brazil \\ ${ }^{3}$ Faculdade de Ciências Biológicas e da Saúde, Universidade Federal dos Vales do Jequitinhonha e Mucuri (UFVJM), Diamantina, \\ Minas Gerais, Brazil \\ ${ }^{4}$ Laboratório de Vibrações Mecânicas e Práticas Integrativas, Departamento de Biofísica e Biometria, Instituto de Biologia Roberto \\ Alcântara Gomes e Policlínica Américo Piquet Carneiro, Universidade do Estado do Rio de Janeiro, Rio de Janeiro, RJ, Brazil \\ ${ }^{5}$ Faculdade de Ciências Agrárias, Universidade Federal dos Vales do Jequitinhonha e Mucuri (UFVJM), Diamantina, \\ Minas Gerais, Brazil \\ ${ }^{6}$ Istituto Auxologico Italiano, IRCCS, Division of Auxology and Metabolic Diseases, Verbania, Italy \\ ${ }^{7}$ School of Sport, Exercise \& Nutrition, Massey University, New Zealand \\ ${ }^{8}$ MATIM, Moulin de la Housse, Université de Reims Champagne Ardenne, Reims Cedex 2 51687, France
}

Correspondence should be addressed to Redha Taiar; redha.taiar@univ-reims.fr

Received 29 June 2021; Revised 5 November 2021; Accepted 11 November 2021; Published 2 December 2021

Academic Editor: Changho Song

Copyright ( $) 2021$ Ana Carolina Coelho-Oliveira et al. This is an open access article distributed under the Creative Commons Attribution License, which permits unrestricted use, distribution, and reproduction in any medium, provided the original work is properly cited.

Objective. Rheumatoid arthritis (RA) causes progressive changes in the musculoskeletal system compromising neuromuscular control especially in the hands. Whole-body vibration (WBV) could be an alternative for the rehabilitation in this population. This study investigated the immediate effect of WBV while in the modified push-up position on neural ratio (NR) in a single session during handgrip strength (HS) in women with stable RA. Methods. Twenty-one women with RA (diagnosis of disease: \pm 8 years, erythrocyte sedimentation rate: \pm 24.8 , age: $54 \pm 11$ years, BMI: $28 \pm 4 \mathrm{~kg} \cdot \mathrm{m}^{-2}$ ) received three experimental interventions for five minutes in a randomized and balanced cross-over order: (1) control—seated with hands at rest, (2) sham-push-up position with hands on the vibration platform that remained disconnected, and (3) vibration-push-up position with hands on the vibration platform turned on $\left(45 \mathrm{~Hz}, 2 \mathrm{~mm}, 159.73 \mathrm{~m} \cdot \mathrm{s}^{-2}\right)$. At the baseline and immediately after the three experimental interventions, the HS, the electromyographic records (EMGrms), and range of motion (ROM) of the dominant hand were measured. The NR, i.e., the ratio between EMGrms of the flexor digitorum superficialis (FDS) muscle and 
HS, was also determined. The lower NR represented the greater neuromuscular efficiency (NE). Results. The NR was similar at baseline in the three experimental interventions. Despite the nonsignificance of within-interventions $(p=0.0611)$ and interaction effect $(p=0.1907)$, WBV exercise reduced the NR compared with the sham and control $\left(p=0.0003, F=8.86, \eta^{2}=\right.$ 0.85 , power $=1.00)$. Conclusion. Acute WBV exercise under the hands promotes neuromuscular modifications during the handgrip of women with stable RA. Thus, acute WBV exercise may be used as a preparatory exercise for the rehabilitation of the hands in this population. This trial is registered with trial registration 2.544.850 (ReBEC-RBR-2n932c).

\section{Introduction}

Rheumatoid arthritis (RA) is a chronic disease that causes progressive damage to the musculoskeletal system. RA compromises neuromuscular control especially in the hands [1-4]. It usually affects the joints in a symmetrical way, thus determining a decline in muscle strength and a progressive reduction of hand functional abilities [5]. Currently, the prevalence of RA is $1-2 \%$ of the world's population, with the rate being two to three times higher in women [6] aged between 20 and 65 years [7].

During the pathological process of RA, the individual may have trouble while performing daily tasks induced by pain, stiffness, and deterioration of the joint structure and function. Damage to musculoskeletal tissue caused by RA's inflammation interferes directly with mobility, generation of muscle strength and neuromuscular control $[8,9]$. Neuromuscular control is necessary and essential for most daily tasks [10]. Thus, women with RA tend to use higher levels of neuromuscular activation in daily tasks than healthy women [11].

Exercise programs are commonly used to improve hand structure and function, but the disparity in determining the most effective exercise for this population remains inconclusive [11]. Whole-body vibration (WBV) exercise is a neuromuscular stimulus method, which can represent a preparatory exercise in promoting lower joint impact and greater neuromuscular modifications [12].

In the context of rehabilitation of patients with stable $\mathrm{RA}$, it is noteworthy that earlier studies evaluating the effect of WBV focused on the lower limbs $[3,13]$. Moreover, few investigations have targeted the upper body by using a static modified push-up position to assess the effects of WBV on healthy participants $[14,15]$. Other studies have examined the effect of WBV exposure on neuromuscular activity of the flexor digitorum superficialis (FDS) muscle $[15,16]$, which is essential for manual skills involving handgrip [17].

Some of the possible mechanisms that may explain the positive effects of WBV exercise are represented by the changes provided in the neuromuscular activation pattern in favor of greater neuromuscular modifications [18]. As evidenced by a two-fold increase in biceps brachial electromyography (EMG) from WBV compared to baseline values [18], it was suggested that this type of treatment can stimulate the neuromuscular system and improve the neuromuscular modifications, i.e., the ratio between EMG and mechanical power, during handgrip activities [19].

From our previous work that reported immediate effects of WBV exercise in promoting a transient increase in muscle contractile performance in untrained healthy women [19, $20]$, it seems plausible that vibration WBV may be a useful preparatory therapy capable of promoting neuromuscular modifications for the rehabilitation of the hands in women with stable RA. Therefore, the objective of the current study was to investigate the effect of WBV exercise in a single session on the static modified push-up position on the handgrip neuromuscular modifications in women with stable RA. As secondary outcomes, we aimed to evaluate the handgrip strength (HS) and concomitant neuromuscular electrical activity of the FDS muscle and determine if the wrist flexion-extension range of motion (ROM) of the dominant hand was enhanced. It was hypothesized that the acute exposure to WBV directly under the hands would promote an increase in wrist flexion-extension ROM and HS, in addition to a concomitant reduction in electromyography (EMGrms).

\section{Materials and Methods}

2.1. Study Design and Participants. Twenty-six women diagnosed with RA were initially screened for eligibility. Twentyone $(n=21)$ eligible RA women were enrolled the study (Figure 1).

The study design was a crossover clinical trial (i.e., all volunteers performed all three experimental interventions in a randomized order) with seven balanced blocks, three by three. Experimental interventions were randomized by sortition, and the participants were blinded. The interventions were performed over a one-week period with a 48hour recovery following each intervention. The familiarization session was performed 48 hours prior to the beginning of the experimental interventions and included a physical examination, anthropometric measurements (height and body mass), and familiarization with the vibrating platform, HS, EMGrms, and ROM. On the day of familiarization, a blood sample was also performed to check the inflammatory activity of the disease through the erythrocyte sedimentation rate (Figure 2 ).

This study was conducted in accordance with the checklists for randomized controlled and clinical trials CONSORT and SPIRIT and ethical principles for research involving human subjects (principles of the Declaration of Helsinki). The study received approval from the Ethics Committee of the Universidade Federal dos Vales do Jequitinhonha $e$ Mucuri (No. 2.544.850) and was submitted to the Registry of Clinical Trials (ReBEC) (RBR-2n932c).

The participants were recruited between March of 2018 and May of 2019, at the medical clinic by the rheumatologists of the Regional Polyclinic, Basic Health Units and radio advertising in Diamantina, MG, Brazil. The inclusion criteria were as follows: women aged between 20 and 70 years, with confirmed diagnosis of RA by a rheumatologist according to the criteria of the American College of Rheumatology 


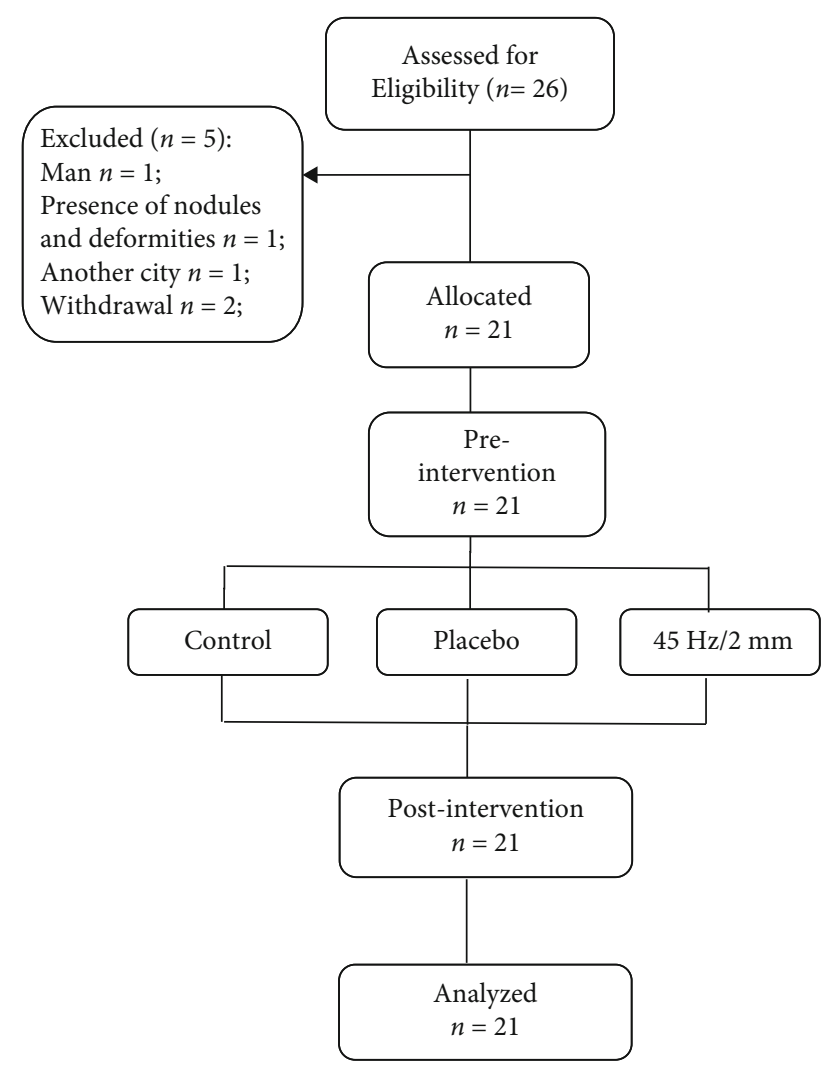

Figure 1: Flow of participants through the study.

[21]. Participants were ineligible, if they presented the following: sensory disturbances; active infections; alcohol or drug abuse; pregnancy or breastfeeding; anticoagulant treatment; any concomitant disease that would prevent the execution of the experimental interventions; any other rheumatological disease; serious complications of RA; nonstable disease; and intra-articular infiltrations or other procedures, such as physical therapy or corrective surgeries, and some contraindication vibrating platform. Participants were advised to avoid adjusting their drug therapy and the use of analgesics for pain.

\subsection{Intervention}

2.2.1. Experimental Interventions. All the volunteers performed the three experimental interventions at the same time of each day, in a controlled thermoneutral environment (means of $22 \pm 1^{\circ} \mathrm{C}$ and $53 \pm 2 \%$ relative humidity).

(1) Control. Participants remained rested for five minutes in a seated position with feet on the floor and hands in the supine position on the lower limbs. There was no WBV stimulus (Figure 3(a)).

(2) Sham. The participants were positioned for five minutes continuously in the push-up position with their hands apart at a distance of $28 \mathrm{~cm}$ on the vibrating platform that was disconnected, but with a sonorous stimulus mimicking the WBV (Figure 3(b)).
(3) Vibration. The participants were positioned for five minutes continuously in the push-up position with their hands apart at a distance of $28 \mathrm{~cm}$ on the vibrating platform turned on, using the vibratory stimulus intensities of $45 \mathrm{~Hz}$, $2 \mathrm{~mm}$, and $159.73 \mathrm{~ms}^{-2}$. The vibrating platform (FitVibe, GymnaUniphy NV, Bilzen, Belgium) produced vertical sinusoidal vibrations resulting in a simultaneous and symmetrical movement on both sides of the body during exposure. A horizontal bar at shoulder height was used to avoid trunk flexion during the intervention and to guarantee an elbow flexion of $10^{\circ}$ (Figure 3(b)). The WBV parameters of frequency $(45 \mathrm{~Hz})$, amplitude $(2 \mathrm{~mm})$, and exposure duration of five minutes were selected in accordance with previous studies reporting positive outcomes [19, 20, 22, 23].

2.3. Procedures. Prior to all three experimental interventions, each participant rested for fifteen minutes in a seated position with their hands placed in a supine position on the lower limbs. This verified the resting electromyography of the FDS muscle. Thereafter, each participant was positioned in one of the experimental interventions described previously. At baseline and immediately after the intervention, the muscle performance of the dominant hand was evaluated using the HS dynamometer (Jamar, Warrenville, USA). The electromyographic record of the FDS muscle of the dominant hand was simultaneously recorded using a portable electromyography data log instrument (Miotec, Porto Alegre, Brazil). Following the ROM of the wrist, flexion-extension of the dominant hand was measured using a universal goniometer (Fibra Cirúrgica, Joinville, Brazil). All the evaluations were performed by a single blinded researcher.

\subsection{Outcome Measures}

2.4.1. Handgrip Strength (HS). Participants were seated with feet on the floor, with the arm in adduction and elbow flexed at $90^{\circ}$, forearm in a neutral position, and wrist extension between $0^{\circ}$ and $30^{\circ}$. The dominant hand performed three repetitions of 3 -second maximum HS. There was a 60 second recovery period between repetitions. HS was determined by the average of the three peak values [24].

2.4.2. Electromyography (EMGrms). Electromyography of the FDS muscle of the dominant hand was recorded using a one-channel portable electromyography. Two passive Ag/ AgCl electrodes (Meditrace, Ludlow Technical Products, Gananoque, Canada) were positioned on the muscle belly of the FDS muscle with a fixed distance of $20 \mathrm{~mm}$, arranged perpendicular to the direction of muscle fibers. One ground electrode was attached to the lateral epicondyle of the humerus according to the position described by SENIAM (Surface Electromyography for the Non-Invasive Assessment of Muscles) [25]. The recorded signals were treated with $10-480 \mathrm{~Hz}$ band pass butterworth filters for signal amplitude analysis and to avoid noise. The analog-todigital conversion of the signals was performed with a 14bit input $\mathrm{A} / \mathrm{D}$ hardware resolution, sampling frequency of $2 \mathrm{kHz}$, common rejection module greater than $100 \mathrm{~dB}$, signal-to-noise ratio less than $3 \mu \mathrm{V}$, and system impedance 


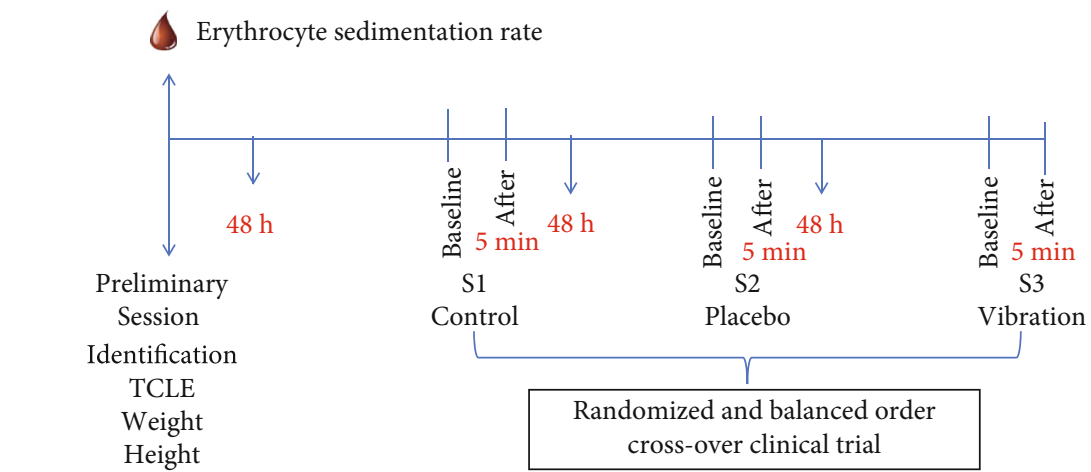

Familiarization (SODA,

HS, EMGrms, ROM,

WBV)

HS: Handgrip strength

EMGrms: Electromyographic ativity of the flexor digitorium superficial muscle ROM: Range of motion of the wrist (flexion and extension)

Parecer Comitê de Ética: 2.544 .850

Registro: ReBEC - RBR-2n932c

Figure 2: Study design.

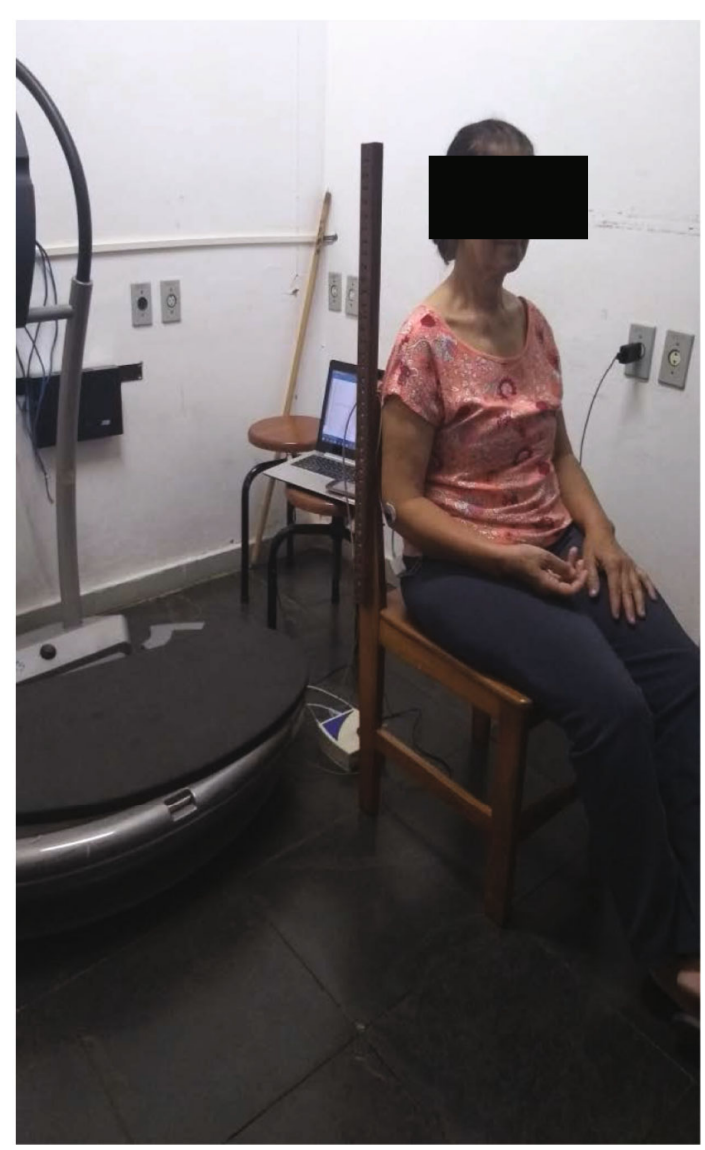

(a)

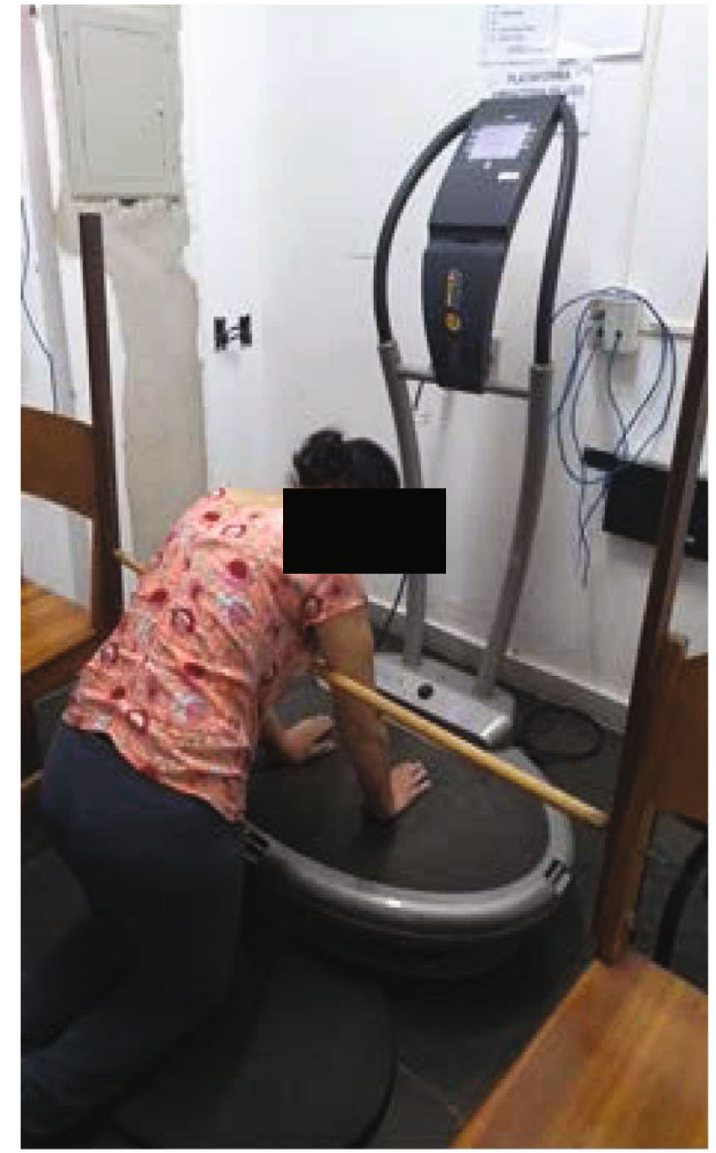

(b)

FIGURE 3: Experimental intervention positions. (a) Control position and (b) push-up position adopted during sham or WBV exercise interventions. 
of 109 Ohms. The signal was captured by surface-active differential sensors and recorded as the Root Mean Square (RMS); this is a quantitative indicator in the recruitment of motor units, in $\mu \mathrm{V}$ and the mean frequency in $\mathrm{Hz}$ [26]. The electromyography signals were collected in $\mu \mathrm{V}$, normalized by peak (peak-to-peak) and transformed into \% RMS by software (MiotecSuite 1.0.1065) for data analysis [25].

Both HS and EMGrms were determined concomitantly by the average of the three repetitions performed before and after the experimental interventions. The HS frequency was $3000 \mathrm{~ms}$ (i.e., $3 \mathrm{~s}$ ) with the EMGrms analyzed using a sliding window of within the interval range of 1000-2000 ms.

2.4.3. Neural Ratio (NR). NR was calculated from the EMGrms of FDS divided by the mechanical power (HS). A lower NR represented greater neuromuscular modification [18].

2.4.4. Range of Motion (ROM). The ROM was measured in degrees from a universal manual goniometer, by a trained researcher. The fixed arm of the goniometer was placed parallel to the longitudinal axis of the proximal end. The movable arm was positioned parallel to the longitudinal axis of the distal end, with the fulcrum at the axis of the joint. Measurements were made of wrist flexion and extension of the dominant hand [27].

\section{Data Analysis}

Data were reported as the mean $\pm 95 \%$ confidence intervals (CIs). Intraclass correlation coefficients assessed the testretest reliability of the HS and electromyography measures. Shapiro-Wilk's test determined normality, and Levene for homogeneity revealed that the data was normally distributed and homogeneous.

The effects of the interventions were compared by splitplot arrangement in a randomized block design and Tukey's (statistical significance level was set at 5\%) test for means comparison (within-test, between-test, and interaction). Thus, the within-test column represented the time factor. The between-test column represented the intervention factor. The interaction column represented the interaction (time $\mathrm{x}$ intervention). The effect size (eta squared: $\eta^{2}$ ) were based on the following criteria: $<0.25$ represented small effect; between 0.25-0.4, moderate effect; and >0.4, large effect [28].

3.1. Sample Size. The sample size was calculated using the GPower ${ }^{\circledR}$ software (Franz Faul, Universitat Kiel, Germany). A sample size of eighteen participants was required for an error probability set at $5 \%$, a power of $80 \%$, and an effect size of 0.64 this was obtained from a previous work evaluating the dose-response of acute WBV exercise in the push-up position on neural ratio in untrained healthy women [19]. Nevertheless, we considered an attrition rate of $15 \%$; the sample size had twenty-one participants ( 7 blocks of $3 \times 3$ participants). There was no participant dropout; therefore, it was not necessary to analyze the data by intention to treat.
TABle 1: Characteristics of participants.

\begin{tabular}{lc}
\hline Characteristic $(n=21)$ & Mean $(95 \% \mathrm{CI})$ \\
\hline Age $(\mathrm{yr})$ & $54(48.99-59.01)$ \\
Body mass $(\mathrm{kg})$ & $72.9(66.98-78.82)$ \\
Height $(\mathrm{m})$ & $1.59(1.56-1.62)$ \\
BMI* $\left.^{*} \mathrm{~kg} / \mathrm{m}^{2}\right)$ & $28.6(26.69-30.51)$ \\
Diagnostic time $(\mathrm{yr})$ & $8(5.36-10.64)$ \\
Erythrocyte sedimentation rate $(\mathrm{mm} / \mathrm{h})$ & $24.8(18.43-31.17)$ \\
SODA $^{*}$ & $106(104.97-107.03)$ \\
\hline
\end{tabular}

BMI*: Body Mass Index; SODA*: Sequential Occupational Dexterity Assessment for Patients with Rheumatoid Arthritis.

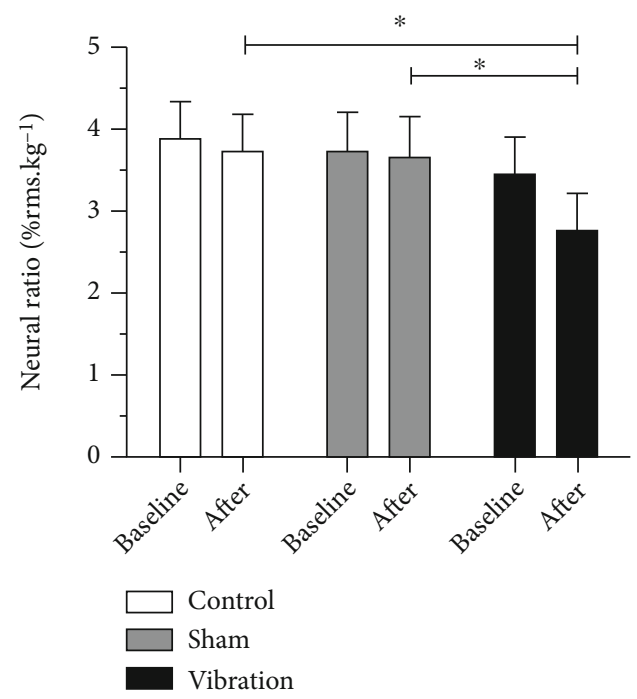

FIgURE 4: Neural ratio at baseline and after the experimental interventions. ${ }^{*} p<0.05$.

\section{Results}

The ICC test-retest reliability of HS, EMG activity of the FDS muscle, and ROM were 0.984, 0.778, and 0.899, respectively.

4.1. Characteristics of Participants. Table 1 presents the volunteers characteristics concerning age, anthropometric parameters, identification of the medical diagnosis's period, and erythrocyte sedimentation rate test to verify the inflammatory activity disease.

\subsection{Primary Outcome}

4.2.1. NR. NR was similar in the three experimental interventions at baseline [baseline-sham: 3.68 (95\% CI: 2.674.68), control: 3.88 (95\% CI: $2.91-4.85$ ), and vibration: 3.42 (95\% CI: 41-4.43)]. Despite no within-interventions $\left(p=0.0611, F=3.94, \eta^{2}=0.66\right.$, power $\left.=0.99\right)$ and interaction $\left(p=0.1907, F=1.69, \eta^{2}=0.50\right.$, power $\left.=0.96\right) \quad$ effect, between-interventions analyses $\left(p=0.0003, F=8.86, \quad \eta^{2}=\right.$ 0.85 , power $=1.00$ ) showed that acute WBV exercise reduced the NR compared with the sham and control 


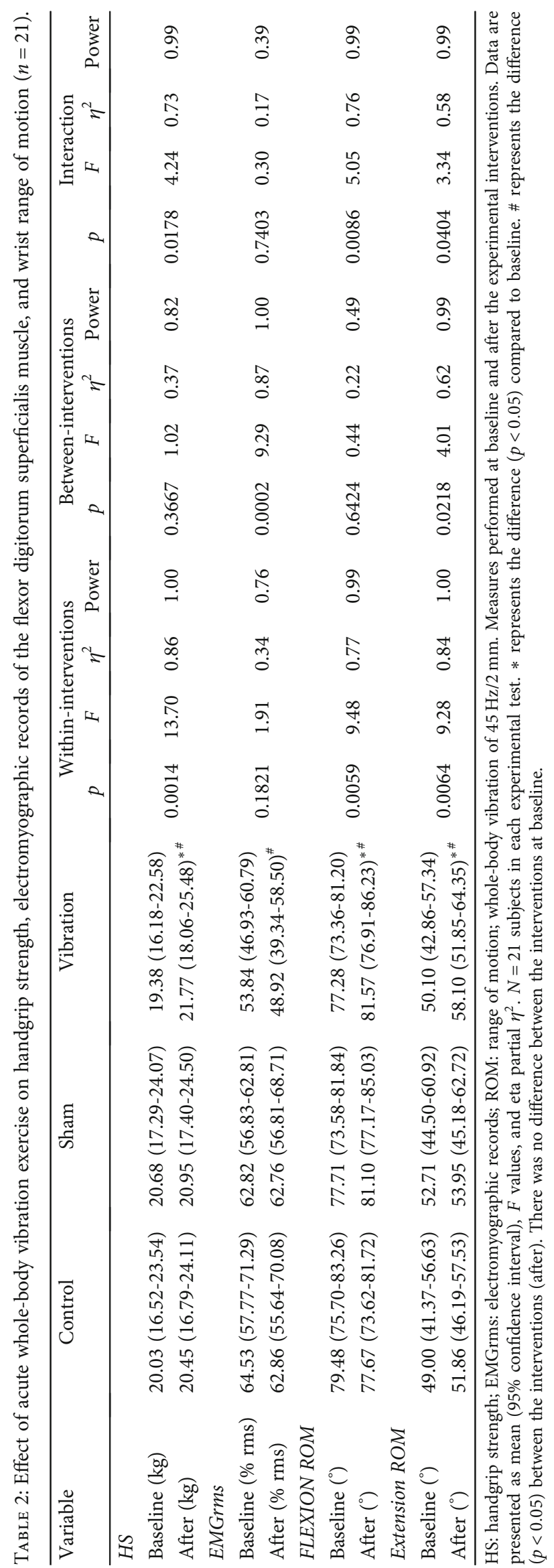


[after-sham: 3.63 (95\% CI: 2.58-4.67), control: 3.71 (95\% CI: 2.74-4.68), and vibration: 2.74 (95\% CI: 1.79-3.69)] (Figure 4).

\subsection{Secondary Outcomes}

4.3.1. HS. HS was similar in the three experimental interventions at baseline. Despite no between-interventions effect, there was interaction effect and within-interventions analyses showed that immediately after the WBV exercise, the HS augmented was approximately 11\% compared with the baseline and other experimental interventions (sham and control) (Table 2).

4.3.2. EMGrms. EMGrms was similar in the three experimental interventions at baseline. Despite no within-interventions and interaction effect, the between-interventions analyses demonstrated that acute WBV exercise decreased significantly the EMGrms activity of FDS muscle compared with sham and control (Table 2).

4.3.3. ROM. Wrist flexion-extension ROM was similar in the three experimental interventions at baseline. Within and between-interventions and interaction analyses showed that acute WBV exercise increased both wrist ROM compared with baseline and the other experimental interventions (sham and control) (Table 2).

\section{Discussion}

The current findings suggest that acute WBV exercise directly under the hands promotes favorable handgrip neuromuscular modifications in women with stable RA. This is in agreement with de Souza et al. [19], who reported that in healthy individuals, the push-up position performed on WBV machine promoted an acute positive effect on HS accompanied by a lower index of neural efficiency, providing a better efficiency of muscle contraction. Thus, WBV represents a possibility of preparatory activity with immediate effect to be used prior to rehabilitation session of stable RA patients. Therefore, to understand the neuromuscular modifications provided by this exercise in this population, first, the effects of acute WBV exercise on HS muscular performance concomitant with the neuromuscular activity of the FDS muscles require consideration.

The HS of the current participants was $20.03 \mathrm{~kg}$ at baseline in all experimental interventions, representing $62 \%$ of the predicted HS in middle-aged and elderly Brazilians. Thus, the disease resulted in a 33 to $37 \%$ impact on HS $[29,30]$. Considering the measurement properties of the HS assessment, the minimum clinically important difference (MCID) scores for women with carpometacarpal osteoarthritis, a chronic disease which results in deterioration of the joint surfaces bone reformation such as RA, are approximately $0.84 \mathrm{~kg}$ (affected side) and $1.12 \mathrm{~kg}$ (unaffected side) $[30,31]$. Although the MCID was estimated for another chronic disease group, the score obtained in our study was $2.39 \mathrm{~kg}$ (1.88-2.90) with WBV, suggesting an important clinical change. This is in agreement with Brorsson et al. [11], who found that patients suffering from arthritis are weaker than healthy individuals in terms of flexion-extension strength. However, our results, as well as those of Villafañe et al. [31] and Speed and Campbell [32], showed that increases of muscle strength in individuals with RA may be due to neural adaptation and, consequently, greater efficiency elicits motor unit activation. Experiments with surface electromyography showed that women with RA tend to use higher levels of neuromuscular activation in daily tasks than healthy women [11], especially during manual skills involving handgrip. In the present study, we opted to evaluate the EMGrms of the FDS muscle. This decision was based on the major muscle group which is responsible for handshake activity since it helps to provide balance for the finger flexion arc $[15,16,33]$. Moreover, we decided for the static push-up position on the vibration platform, as there is evidence of greater muscle activation in the upper limb muscles during acute WBV stimulation of this position [34]. However, in our study, the acute WBV stimulation reduced muscle activation levels immediately after the WBV intervention, suggesting that fewer motor units were required to perform the same handgrip activity.

According to our findings, a single acute WBV exercise session directly under the hands, in a modified static pushup position, was able to promote neuromuscular changes in handgrip in women with RA. We observed that the NR of the participants was about $3.66 \%$ at the baseline in all experimental interventions. After exposure to the vibration intervention, there was a reduction of approximately $24.5 \%$ in handgrip NR compared to sham and control interventions. These results demonstrate that a single session of acute WBV, directly under the hands, promotes greater neuromuscular modifications. Corroborating the results of de Souza et al. [19], which suggested an acute dose-dependent WBV stimulus in a static push-up position potentiates handgrip myogenic response; additionally, the mechanism underlying this positive effect seems to be related to the stimulation of the neuromuscular system and subsequent postactivation potentiation, leading to neural enhancement.

Although there are few publications in the context of the rehabilitation of patients with stable RA involving WBV and upper limbs. It is noteworthy that studies generally evaluated the effect of training with WBV, focused on the lower limbs $[3,13,35]$. Regarding the changes and consequent neuromuscular modifications of the hands, to our knowledge, there are no studies that have reported this in RA population. However, Krol et al. [34] and other researches demonstrated an increase in the neuromuscular efficiency and concluded that vibration exercise can be useful to stimulate the neuromuscular system in healthy population $[14,19,20,36]$.

The current findings support the concept that the acute WBV exercise potentiates the neural response. The following protocol description reproduces information already reported elsewhere $[15,19]$. WBV exercise is reported to represent an alternative exercise for the treatment of RA due to its ability in promoting lower joint impact and greater neuromuscular modifications. Previous studies have reported satisfactory results of using WBV training that ranged from $24 \mathrm{~Hz}, 2 \mathrm{~mm}$, acceleration $\left[45.43 \mathrm{~m} \cdot \mathrm{s}^{-2}\right]$ to 
$30 \mathrm{~Hz}, 3 \mathrm{~mm}$, acceleration $\left[106.48 \mathrm{~m} \cdot \mathrm{s}^{-2}\right]$. Moreover, the stimulus duration varied from 10.5 minutes to 15 minutes intermittently $[13,35,36]$. Nevertheless, the current WBV parameters (frequency: $45 \mathrm{~Hz}$; amplitude: $2 \mathrm{~mm}$, acceleration: $159.73 \mathrm{~m} \cdot \mathrm{s}^{-2}$ ) of 5 minutes continuous WBV were selected based on previous research $[19,20,22,23]$. Additionally, all participants adhered to the 5-minute protocol and successfully completed the vibration without discomfort.

In the context of joint damage in patients with RA, one of the most affected is the wrist (78\%) [37]. Thus, usually, a hand's joints present reduction in muscle contraction, firing rate of motor units, ROM, and mobility, as well as change in the muscle fiber type [38]. In the current study, the wrist flexion ROM in the RA group was on average $11.8^{\circ}$ lower than normal values [27], and after acute WBV exposure, there was an increase around $4.29^{\circ}$ (compared with a sham test showing an increase around $3.39^{\circ}$ ). Regarding the wrist extension ROM, the RA group presented a $19.4^{\circ}$ value lower than the predicted values [27], which increased after acute WBV (compared with sham test showing an increase around $4.15^{\circ}$ ).

The rationale for WBV exercise as a preparatory activity before training or rehabilitation sessions is based on the premise of promoting "active muscle warm-up" [22, 23]. Active warm-up consists of low-intensity movements that are effective in raising body temperature, promoting tissue warm-up, and producing a variety of improvements in physiological functions [39]. Therefore, warm-up activities are necessary to prepare the body for vigorous physical activity since they increase performance and decrease the risk of muscle injury. Moderate intensity of active warming and passive warming can increase muscle performance by 3 to $9 \%$ [39]. In addition, WBV exercise is purported to increase neuromuscular spindle activity, triggering a reflex-stretch response [40], and consequently creates a small and rapid change in muscle length [41].

In the literature, few studies have investigated the effects of acute WBV on ROM that have focused mainly on the lower limb flexibility [42, 43]. According to Oliveira et al. [44], joint ROM is related to functionality and is a determinant factor of morbidity and a mortality predictor in RA patients. Thus, we considered relevant to investigate the effect of acute WBV exercise applied directly to the hand on the ROM of the wrist flexion-extension. The current data suggests that vibratory exercise significantly improved the wrist ROM, probably triggering small and rapid modifications in muscle length. However, this requires further investigation to substantiate this proposition.

Inevitably, this study had some inherent limitations. As this investigation was only performed with RA women, a certain degree of caution should be acknowledged. However, the statistical analyses demonstrated a large effect size within-between-interventions, as well as interaction for NR. The blood analysis of Erythrocyte Sedimentation Rate and the SODA instrument demonstrated that the studied population was not in the inflammatory activity phase of the disease and presented satisfactory manual dexterity. Moreover, because specific conditions were evaluated, such as platform position, stimulus duration, frequency, and amplitude, and EMGrms analyses of only one muscle group; therefore, the findings of this study cannot be extrapolated to other parameters of acute WBV and cannot be generalized to another population.

\section{Conclusions}

In conclusion, acute WBV exercise, directly under the hands, in the push up position, promotes neuromuscular modifications, suggesting positive impact on neuromuscular performance and wrist ROM, with concomitant reduction in handgrip NR in women with stable RA. As clinical relevance, acute WBV exercise under the hands of stable RA patients suggests positive effects on aspects of structure and function related to manual activities that involve object manipulation. Thus, acute WBV exercise may be a complementary and alternative preparatory exercise for the treatment of patients with musculoskeletal dysfunction.

\section{Data Availability}

The datasets used and/or analyzed during the current study are available from the corresponding author on reasonable request.

\section{Conflicts of Interest}

The authors declare that there is no conflict of interest regarding the publication of this paper.

\section{Authors' Contributions}

AC, AL, VA, and ACR conceived and designed the experiments. AC, AL, and LM performed the experiments. AC, LM, SF, JM, VR, PH, HR, VA, FM, RG, DC, MB, RT, AS, $\mathrm{VL}$, and ACR analyzed the data (interpreted results of experiments, prepared figures, and drafted, edited, and revised the manuscript). AC, SF, JS, HR, VA, and ACR contributed the materials/analysis tools. All authors read and approved the manuscript.

\section{Acknowledgments}

The authors would like to thank the Universidade Federal dos Vales do Jequitinhonha e Mucuri for the institutional support and the Conselho Nacional de Desenvolvimento Científico e Tecnológico (CNPq), the Coordenação de Aperfeiçoamento de Pessoal de Nível Superior (CAPES), and FAPEMIG for the support and scholarships. We acknowledge MSc. Ana Carolina Coelho de Oliveira (2019) who provided data from her master's degree conducted in the Universidade Federal dos Vales do Jequitinhonha e Mucuri, Minas Gerais, Brazil.

\section{References}

[1] A. Garg, the WISTAH Hand Study Team, K. T. Hegmann et al., "The WISTAH hand study: a prospective cohort study of distal upper extremity musculoskeletal disorders," $B M C$ Musculoskeletal Disorders, vol. 13, no. 1, 2012. 
[2] G. Plasqui, "The role of physical activity in rheumatoid arthritis," Physiology \& Behavior, vol. 94, no. 2, pp. 270275, 2008.

[3] A. Prioreschi, M. Tikly, and J. A. McVeigh, "A three month controlled intervention of intermittent whole body vibration designed to improve functional ability and attenuate bone loss in patients with rheumatoid arthritis," BMC Musculoskeletal Disorders, vol. 15, no. 1, 2014.

[4] O. Sangha, "Epidemiology of rheumatic diseases," Rheumatology (Oxford), vol. 39, supplement 2, pp. 3-12, 2000.

[5] M. I. Corbacho and J. J. Dapueto, "Assessing the functional status and quality of life of patients with rheumatoid arthritis," Revista Brasileira de Reumatologia, vol. 50, no. 1, pp. 31-43, 2010.

[6] L. M. da Mota, I. M. Laurindo, and L. L. dos Santos Neto, "Demographic and clinical characteristics of a cohort of patients with early rheumatoid arthritis," Revista Brasileira de Reumatologia, vol. 50, no. 3, pp. 235-240, 2010.

[7] M. Adawi, S. Firas, and A. Blum, "Rheumatoid arthritis and atherosclerosis," The Israel Medical Association Journal, vol. 21, no. 7, pp. 460-463, 2019.

[8] J. Poole, "Hand function in rheumatoid arthritis," in Hand Function, pp. 73-82, Springer, Cham, 2019.

[9] R. Iop, A. Paula Shiratori, L. Ferreira, N. Gomes Borges Júnior, S. Domenech, and M. Silva Gevaerd, "Production capacity of maximal isometric grip strength in women with rheumatoid arthritis: a pilot study," Fisioterapia e Pesquisa, vol. 22, pp. 11-16, 2015.

[10] M. A. Bianchin, G. Paula, M. P. Carvalho, R. Acayaba, and C. RJMR, "Manual de orientações de terapia ocupacional quanto à proteção articular para pacientes com Artrite Reumatóide," Medicina de Reabilitação, vol. 29, pp. 23-28, 2010.

[11] S. Brorsson, A. Nilsdotter, C. Thorstensson, and A. Bremander, "Differences in muscle activity during handdexterity tasks between women with arthritis and a healthy reference group," BMC Musculoskeletal Disorders, vol. 15, no. 1, p. 154, 2014.

[12] M. M. Alam, A. A. Khan, and M. Farooq, "Effect of wholebody vibration on neuromuscular performance: a literature review," Work, vol. 59, no. 4, pp. 571-583, 2018.

[13] A. Prioreschi, M. A. Makda, M. Tikly, and J. A. McVeigh, "In patients with established RA, positive effects of a randomised three month WBV therapy intervention on functional ability, bone mineral density and fatigue are sustained for up to six months," PLoS One, vol. 11, no. 4, article e0153470, 2016.

[14] R. Di Giminiani, L. Fabiani, G. Baldini, G. Cardelli, A. Giovannelli, and J. Tihanyi, "Hormonal and neuromuscular responses to mechanical vibration applied to upper extremity muscles," PLoS One, vol. 9, no. 11, article e111521, 2014.

[15] Z. Ashnagar, A. Shadmehr, M. Hadian, S. Talebian, and S. Jalaei, "The effects of whole body vibration on EMG activity of the upper extremity muscles in static modified push up position," Journal of Back and Musculoskeletal Rehabilitation, vol. 29, no. 3, pp. 557-563, 2016.

[16] D. Morel, P. Marín, E. Moreira-Marconi, C. Dionello, and M. Bernardo-Filho, "Can whole-body vibration exercises in different positions change muscular activity of upper limbs?," A Randomized Trial. Dose-Response, vol. 16, 2018.

[17] C. Long 2nd, P. W. Conrad, E. A. Hall, and S. L. Furler, "Intrinsic-extrinsic muscle control of the hand in power grip and precision handling. An electromyographic study," The Journal of
Bone and Joint Surgery. American Volume, vol. 52, no. 5, pp. 853-867, 1970.

[18] C. Bosco, M. Cardinale, and O. Tsarpela, "Influence of vibration on mechanical power and electromyogram activity in human arm flexor muscles," European Journal of Applied Physiology and Occupational Physiology, vol. 79, no. 4, pp. 306-311, 1999.

[19] A. L. C. de Souza, V. A. Mendonça, A. C. C. de Oliveira et al., "Whole body vibration in the static modified push-up position in untrained healthy women stimulates neuromuscular system potentiating increased handgrip myogenic response," Journal of Bodywork and Movement Therapies, vol. 24, no. 4, pp. 233-238, 2020.

[20] L. M. M. Santos, A. C. C. Oliveira, S. F. Fonseca et al., "Wholebody vibration exercise in different postures on handgrip strength in healthy women: a cross-over study," Frontiers in Physiology, vol. 11, 2021.

[21] D. Aletaha, J. Funovits, and J. S. Smolen, "Physical disability in rheumatoid arthritis is associated with cartilage damage rather than bone destruction," Annals of the Rheumatic Diseases, vol. 70, no. 5, pp. 733-739, 2011.

[22] D. J. Cochrane, S. R. Stannard, E. C. Firth, and J. Rittweger, "Comparing muscle temperature during static and dynamic squatting with and without whole-body vibration," Clinical Physiology and Functional Imaging, vol. 30, no. 4, pp. 223229, 2010.

[23] D. J. Cochrane, S. R. Stannard, A. J. Sargeant, and J. Rittweger, "The rate of muscle temperature increase during acute wholebody vibration exercise," European Journal of Applied Physiology, vol. 103, no. 4, pp. 441-448, 2008.

[24] E. Fess and C. Moran, Clinical Assessment Recommendations, pp. 6-8, American Society of Hand Therapists, 1981.

[25] H. J. Hermens, B. Freriks, C. Disselhorst-Klug, and G. Rau, "Development of recommendations for SEMG sensors and sensor placement procedures," Journal of Electromyography and Kinesiology, vol. 10, no. 5, pp. 361-374, 2000.

[26] H. Suzuki, R. A. Conwit, D. Stashuk, L. Santarsiero, and E. J. Metter, "Relationships between surface-detected EMG signals and motor unit activation," Medicine and Science in Sports and Exercise, vol. 34, no. 9, pp. 1509-1517, 2002.

[27] C. C. Norkin and D. J. White, Measurement of Joint Motion: A Guide to Goniometry, FA Davis, 2016.

[28] J. Cohen, Statistical Power Analysis for the Behavioral Sciences, Academic press, 2013.

[29] R. Novaes, A. Miranda, J. Silva, B. Tavares, and V. Dourado, "Equações de referência para a predição da força de preensão manual em brasileiros de meia idade e idosos," Fisioterapia e Pesquisa, vol. 16, no. 3, pp. 217-222, 2009.

[30] P. Bobos, G. Nazari, Z. Lu, and J. C. MacDermid, "Measurement properties of the hand grip strength assessment: a systematic review with meta-analysis," Archives of Physical Medicine and Rehabilitation, vol. 101, no. 3, pp. 553-565, 2020.

[31] J. H. Villafañe, K. Valdes, L. Bertozzi, and S. Negrini, "Minimal clinically important difference of grip and pinch strength in women with thumb carpometacarpal osteoarthritis when compared to healthy subjects," Rehabilitation Nursing, vol. 42, no. 3, pp. 139-145, 2017.

[32] C. A. Speed and R. Campbell, "Mechanisms of strength gain in a handgrip exercise programme in rheumatoid arthritis," Rheumatology International, vol. 32, no. 1, pp. 159-163, 2012. 
[33] S. F. Duncan, C. E. Saracevic, and R. Kakinoki, "Biomechanics of the hand," Hand Clinics, vol. 29, no. 4, pp. 483-492, 2013.

[34] P. Krol, M. Piecha, K. Slomka, G. Sobota, A. Polak, and G. Juras, "The effect of whole-body vibration frequency and amplitude on the myoelectric activity of vastus medialis and vastus lateralis," Journal of Sports Science and Medicine, vol. 10, no. 1, pp. 169-174, 2011.

[35] A. Alev, A. Mihriban, E. Bilge et al., "Effects of whole body vibration therapy in pain, function and depression of the patients with fibromyalgia," Complementary Therapies in Clinical Practice, vol. 28, pp. 200-203, 2017.

[36] T. Trans, J. Aaboe, M. Henriksen, R. Christensen, H. Bliddal, and H. J. T. K. Lund, "Effect of whole body vibration exercise on muscle strength and proprioception in females with knee osteoarthritis," The Knee, vol. 16, no. 4, pp. 256-261, 2009.

[37] M. A. J. M. C. Mezzari, J. de Oliveira, S. C. Domenech, N. G. Borges Junior, and M. S. Gevaerd, "Motion range assessment in rheumatoid arthritis: a literature review," Cadernos Brasileiros de Terapia Ocupacional, vol. 25, pp. 387-400, 2017.

[38] K.-S. Lee and M.-C. J. S. Jung, "Ergonomic Evaluation of Biomechanical Hand Function," Safety and Health at Work, vol. 6, no. 1, pp. 9-17, 2015.

[39] N. Avelar, F. Salvador, V. Ribeiro et al., "Whole body vibration and post-activation potentiation: a study with repeated measures," International Journal of Sports Medicine, vol. 35, no. 8, pp. 651-657, 2014.

[40] R. Ritzmann, A. Kramer, M. Gruber, A. Gollhofer, and W. Taube, "EMG activity during whole body vibration: motion artifacts or stretch reflexes?," European Journal of Applied Physiology, vol. 110, no. 1, pp. 143-151, 2010.

[41] D. J. Cochrane, I. D. Loram, S. R. Stannard, and J. Rittweger, "Changes in joint angle, muscle-tendon complex length, muscle contractile tissue displacement, and modulation of EMG activity during acute whole-body vibration," Muscle \& Nerve, vol. 40, no. 3, pp. 420-429, 2009.

[42] J. Cronin, M. Nash, and C. Whatman, "The acute effects of hamstring stretching and vibration on dynamic knee joint range of motion and jump performance," Physical Therapy in Sport, vol. 9, no. 2, pp. 89-96, 2008.

[43] W. A. Sands, J. R. McNeal, M. H. Stone, W. L. Kimmel, G. Gregory Haff, and J. MJEJoSS, "The effect of vibration on active and passive range of motion in elite female synchronized swimmers," European Journal of Sport Science, vol. 8, no. 4, pp. 217-223, 2008.

[44] S. C. G. Oliveira, L. M. Oliveira, A. Jones, and N. JJRBdR, "Isokinetic assesment of ankles in patients with rheumatoid arthritis," Revista Brasileira de Reumatologia, vol. 55, no. 4, pp. 318324, 2015. 